\title{
Aprendizaje basado en juegos (GBL) aplicado a la enseñanza de la matemática en educación superior. Una revisión sistemática de literatura
}

\author{
Sergio A. Zabala-Vargas ${ }^{(1) \star}$, Dayan A. Ardila-Segovia(1), Lewis H. García-Mora(2), Bárbara L. de Benito-Crosetti(3) \\ (1) Facultad de Ingeniería de Telecomunicaciones, Universidad Santo Tomás, Cr 18 \# 9-27, Bucaramanga-Colombia \\ (e-mail: sergio.zabala@ustabuca.edu.co, segovia_96@hotmail.es). \\ (2) Unidad de Desarrollo Curricular y Formación Docente, Cr 18 \# 9-27, Bucaramanga-Colombia \\ (e-mail: lewis.garcia@ustabuca.edu.co). \\ (3) Programa de Doctorado en Tecnología Educativa, Universidad de las Islas Baleares, Carretera de Valldemossa km \\ 7.5, 07122 Palma-España (e-mail: barbara.debenito@uib.es).
}

* Autor a quien debe ser dirigida la correspondencia

Recibido May. 2, 2019; Aceptado Jun. 25, 2019; Versión final Ago. 9, 2019, Publicado Feb. 2020

\begin{abstract}
Resumen
El objetivo de este artículo es presentar una revisión sistemática sobre el aprendizaje basado en juegos, aplicado a la enseñanza de las matemáticas en la educación superior. La tecnología en las aulas ha generado cambios notables y nuevos entornos de aprendizaje, entre los cuales los juegos logran captar la atención de muchos usuarios, generar compromiso y mejores resultados de aprendizaje. Se utilizó una metodología de revisión de registros a partir de cinco índices bibliográficos y bases de datos de interés en el área educacional, se realizó el análisis bibliométrico correspondiente, la evaluación de calidad y la generación de conclusiones. Los resultados muestran 19 registros que abordan diferentes mediaciones y que se orientan al desarrollo cognitivo, emocional, afectivo, de habilidades blandas y de comportamiento. Estos hallazgos permiten a los investigadores interesados en el tema y a las instituciones educativas analizar la relevancia y los aportes positivos de los juegos (digitales o no digitales) en los procesos de enseñanza-aprendizaje.
\end{abstract}

Palabras clave: aprendizaje basado en juegos; motivación; revisión sistemática de la literatura; matemáticas; educación superior.

\section{Game-based learning (GBL) applied to the teaching of mathematics in higher education. A systematic review of the literature}

\begin{abstract}
The goal of this article is to present a systematic revision of the literature about game-based learning, applied to the teaching of mathematics in higher education. Technology in the classrooms has generated notable changes and new learning environments, among which computer games capture the attention of many users, generate compromise and better learning results. Through the methodology of record review from five bibliographic indexes and databases, of interest for the educational area, the corresponding bibliometric analysis, the quality evaluation and the generation of conclusions were done. The results show 19 records that approach different mediations and that are oriented to the cognitive, emotional, affective, soft skills, and behavioral development. These findings allow researchers interested in the topic and higher educational institutions to analyze the relevance and positive contributions of games (digital and non-digital) in the teaching-learning processes.
\end{abstract}

Keywords: game-based learning; motivation; systematic literature review; mathematics; higher education 


\section{INTRODUCCIÓN}

La importancia del desarrollo de competencias y habilidades matemáticas en programas de educación superior es evidente en múltiples áreas de conocimiento. Por ejemplo, en el área de ingeniería, la Organización para la Acreditación de los Programas de Ingeniería en Estados Unidos (Accreditation Board for Engineering and Technology-ABET), comprende a este campo de acción disciplinar como la aplicación de matemáticas y ciencias que permiten determinar el uso de materiales y fuerzas de la naturaleza en claro beneficio del ser humano (ABET, 2016). Este mismo referente resalta la importancia de incorporar en el currículo, las matemáticas, durante un periodo no inferior a un año y medio. De otra parte, la Red Europea para Acreditación en Ingeniería (European Network for Engineering Accreditation-ENAEE), indica que el proceso de enseñanza-aprendizaje para un ingeniero debe permitir demostrar su conocimiento y entendimiento de las matemáticas y otras ciencias básicas subyacentes a la especialización que desarrolle en su programa específico (ENAEE, 2015). En el caso colombiano, por citar un solo ejemplo en Latinoamérica, el Ministerio de Educación Nacional expresa en la Resolución 2773 de Noviembre de 2003, asociada a la definición de las características específicas de calidad para los programas de formación profesional de pregrado en ingeniería que: "El programa debe poseer la fundación teórica y metodológica de la Ingeniería que se fundamenta en los conocimientos de las ciencias naturales y matemáticas...", a su vez resalta que "el área de ciencias básicas está integrada por cursos de ciencias naturales y matemáticas, área sobre la cual radica la formación básica científica del ingeniero" (Ministerio de Educación Nacional de Colombia, 2003).

En otra área de conocimiento, como es el caso de la administración y las finanzas, las matemáticas son herramientas claves para el manejo, cuantificación y proyección de los recursos económicos de una organización, así como en la toma de decisiones empresariales. Como plantean Farias y Pérez (2010) la comprensión de los modelos matemáticos financieros y la motivación que se requiere para la enseñanza de estos, son de interés para esta área del saber. En el ámbito médico, las habilidades matemáticas son importantes para la interpretación de los estudios de laboratorio, entendimiento de la información nutrimental, cálculo y ajustes de dosis de medicamentos, interpretación de los resultados publicados en la bibliografía médica, sustento para la toma de decisiones, uso e interpretación de los riesgos y cálculo de probabilidades. Por tanto, como indica Olmedo y Ariza (2012), es imprescindible que el médico esté consciente de la necesidad de reflexionar sobre su propio desempeño en relación con las habilidades matemáticas, y en caso de requerirlo, buscar apoyo para mejorar tal situación. Finalmente, en el campo de las ciencias naturales, los conocimientos matemáticos son una herramienta para ofrecer respuestas a preguntas problemáticas que surgen en su quehacer. Barquero et al. (2011) indican que es de vital importancia que los estudiantes aprendan a utilizar y a aplicar los conocimientos matemáticos a las situaciones problemáticas típicas con las que se encontrarán en su especialidad científica.

Ahora bien, la enseñanza de la matemática en la educación superior suele tener malas prácticas que afectan los propósitos de esta. Bergeson (2000) cita como elementos más relevantes 1-) Pérdida de significado para los estudiantes y descontextualización. Se evidencia que gran cantidad de estudiantes consideran a las matemáticas como un proceso que se limita a la memorización; y 2-) La linealidad y la formalidad asociada con la enseñanza de matemáticas se obtienen de los esquemas de los libros de texto y tienden a reproducir la aceptación pasiva de las matemáticas desde lo abstracto. En este tipo de procesos de pensamiento existe muy poca relación entre las actividades trabajadas y la vida diaria del estudiante. El mismo Bergeson (2000) presenta aspectos y acciones pedagógicas que pueden mejorar la dinámica de la enseñanza de las matemáticas entre los que se destacan que los estudiantes utilicen ambientes soportados en herramientas computacionales que faciliten la interacción, la realimentación y la autoevaluación, así como el trabajo cooperativo como estrategia de construcción de conocimiento. Este último aspecto también es resaltado por las aproximaciones teóricas del trabajo colaborativo de Johnson et al. (1994) y por la validación experimental realizada por (Lizcano-Dallos et al., 2016). Las competencias docentes, la reflexión sobre nuevas didácticas y los nuevos entornos de aprendizaje son aspectos que, como resaltan (Cabero-Almenara, 2016; Salinas et al., 2014), también influyen en la dinámica de los procesos de enseñanza.

Es en este sentido, donde estrategias pedagógicas innovadoras para la enseñanza de la matemática y nuevos escenarios didácticos deben ser discutidos. Este es el caso de dos conceptos que serán base referencial del desarrollo del presente artículo. El primero de estos, la gamificación, se puede entender como: "El uso de elementos de diseño de juegos en contextos distintos del juego" (Deterding et al., 2011). A su vez, Blohm y Leimeister (2013) indican que los desarrollos y mediaciones pedagógicas realizadas en el tema de gamificación cuentan con muchos elementos comunes, como es el caso de: 1-) las mecánicas (documentación del comportamiento, sistema de puntaje, insignias, rankings, niveles, entre otros); 2-) las dinámicas (Competición, colaboración, exploración, colección, entre otros) y 3-) las motivaciones (Curiosidad intelectual, reconocimiento social, estimulación cognitiva, intercambio social, entre otros). 
El segundo concepto fundamental para el desarrollo de la presente revisión es el Game Based Learning-GBL (Aprendizaje Basado en Juegos). El GBL, en términos sencillos, se entiende como el uso de juegos (y su diseño) en ambientes y con intencionalidades educativas. Steiner et al. (2009) manifiestan en su artículo que la idea central del aprendizaje basado en juegos es utilizar al menos parte del tiempo que las personas dedican a los juegos de computadora para propósitos educativos. Por su parte, Kapp (2012) indica que el GBL facilita el aprendizaje por asentarse sobre el juego: el proceso se sigue más fácilmente mientras se asimilan los conceptos, ya que el juego crea un entorno virtual que recrea situaciones propias de la realidad (simuladores) y de esta forma los usuarios (alumnos) aprenden a desenvolverse en un contexto sin riesgo, pero con normas, interactividad y realimentación.

Complementando lo anterior, Chen y Wang (2009) indican que el aprendizaje basado en juegos generalmente se considera como un medio eficaz para permitir que los alumnos construyan conocimiento jugando, mantengan una mayor motivación y apliquen el conocimiento adquirido para resolver problemas de la vida real. Por lo tanto, el aprendizaje basado en juegos se convierte en un método prometedor para proporcionar situaciones de aprendizaje altamente motivadoras a los estudiantes. A través de una combinación de juegos, resolución de problemas, aprendizaje situado y desafíos, el aprendizaje basado en juegos puede ayudar a los estudiantes a construir conocimiento desde la ambigüedad, la complejidad, la prueba y el error.

Sobre el uso de herramientas computacionales para la enseñanza de la matemática el panorama es bastante amplio. Kebritchi et al. (2010) presentan los efectos que el uso de herramientas computacionales, particularmente los juegos, tienen en los estudiantes de curso de matemáticas. Esta investigación indica que hay una relevancia significativa de los resultados en cuanto a cumplimiento de logros de aprendizaje entre el grupo experimental y el grupo de control, así como un incremento en la motivación de los estudiantes cuando utilizan los juegos en salón de clases y en laboratorios. Adicionalmente en el trabajo presentado en Nah et al. (2014), se evidencia cómo el uso de elementos asociados a los juegos de computadora, permiten fortalecer resultados importantes en un proceso educativo asociado a las matemáticas, como son: Compromiso, participación, motivación, cumplimiento de metas, entre otros.

En la publicación presentada en Huang et al. (2013), los autores revisan la relación de las características de un juego en línea con la motivación de los estudiantes hacia el proceso de aprendizaje. Se logra demostrar con soporte estadístico que para un diseño en GBL es fundamental considerar tres características principales: A-) La estructura (reglas fáciles de seguir, metas claramente presentadas, tareas claras, información clara antes y durante el juego, proveer ayuda para completar las tareas); B-) involucramiento (el juego permite contar con un rol, mantiene el interés, es divertido) y C-) la apariencia (el juego cuenta con gráficas, animaciones y elementos de audio atractivos).

Otro caso que integra el desarrollo de herramientas basadas en la lúdica, recurriendo a realidad aumentada, con trabajo colaborativo basado en ambientes móviles y uso de smartphone es presentado en Ke y Hsu (2015). Particularmente esta experiencia demuestra que el utilizar dispositivos móviles para generar espacios de trabajo colaborativo contribuye a soportar el desarrollo de conocimiento en el aula. La propuesta compartida en Sung et al. (2015) presenta resultados empíricos de la implementación de un juego contextual para facilitar la toma de decisiones en temas educativos. Dos iniciativas con aplicación empírica de elementos de gamificación y de GBL en la potencialización de la motivación y actitud de los estudiantes hacia el proceso de aprendizaje se presentan en (Galbis Córdova et al., 2017; Yildirim, 2017).

En Zabala-Vargas et al. (2013) se relacionan herramientas de modelamiento, simulación y mecánicas de gamificación para mejorar el desempeño académico de los estudiantes de cursos de matemáticas básicas en ingeniería. En los trabajos presentados en Borras et al. (2014), Collazos et al. (2014) y Fonseca-Tovar et al. (2018), se centra la discusión en el uso de herramientas de gamificación trabajo colaborativo en el desarrollo de competencias matemáticas específicas. Otros trabajos con experiencias significativas de incorporación de Aprendizaje Basado en Juegos y gamificación en educación superior se comparten en (Hernández-Horta et al., 2018; Martínez, 2017). Asimismo, se encuentran revisiones sistemáticas como la presentada por Connolly et al. (2012) relacionada al impacto de juegos digitales en la educación, la actualización de este trabajo compartida en Boyle et al. (2016), la revisión realizada en Hamari et al. (2014) y el aporte en el área de la educación superior de (Subhash y Cudney, 2018).

Finalmente, con lo presentado hasta este punto, se evidencia la relevancia para el sector educativo universitario de generar panoramas de las corrientes, estrategias y tendencias orientados al desarrollo de competencias y habilidades matemáticas, siendo esta la motivación de generar una revisión sistemática de la literatura sobre el tema. La revisión sistemática compartida en este artículo aporta, de manera específica, al área de las matemáticas; lo cual no se encuentra, hasta donde los autores pudieron verificar, en la literatura; y puede aportar en el fomento de las investigaciones del sector. 


\section{METODOLOGÍA}

La estrategia utilizada en esta revisión se basa en la adaptación del método propuesto por Petticrew y Roberts (2006), apropiado por Gast et al. (2017), para desarrollar revisiones sistemáticas en las ciencias sociales. Este procedimiento implica varios pasos, los cuales adaptados al interés del presente artículo, se sintetizan en: 1) Formulación de preguntas de investigación para orientar la revisión sistemática de la literatura; 2) Definición de los términos y las ecuaciones de búsqueda, así como la selección de las bases de datos más pertinentes para el área de conocimiento; 3) Formulación de criterios de inclusión y exclusión que permitan delimitar y precisar aún más la búsqueda bibliográfica; 4) Análisis bibliométrico de los artículos producto de la revisión; 5) Evaluación de la calidad científica de las publicaciones obtenidas utilizando criterios de calidad predefinidos, incluyendo en el análisis final de categorías solo aquellos que aprobaron esta evaluación; y 6) Realización de análisis categorial centrado en la tipología de los mecanismos utilizados en los procesos de gamificación y aprendizaje basado en juegos (propósito primario del juego, digital o no digital, genero del juego, plataforma y sub-área), además las salidas de interés pedagógico de los trabajos (dominio afectivo-motivacional, dominio cognitivo de adquisición y compresión y dominio comportamental).

\section{Paso 1. Preguntas de investigación}

Las preguntas de investigación formuladas fueron las siguientes: I) ¿Qué tipos de juegos y qué características se encuentran en la literatura para la enseñanza de las matemáticas en educación superior?; II) ¿Cuáles son las metodologías y los instrumentos de investigación más utilizados en los artículos hallados en la revisión? y III) ¿Cuáles son las principales dimensiones del comportamiento, la motivación y el desempeño de los estudiantes, en las intervenciones publicadas en los artículos obtenidos en la revisión?. Con estas tres preguntas se buscó dar respuesta a la motivación principal del artículo, que se centra en: ¿Cómo influye el uso del aprendizaje basado en juegos, en la enseñanza de la matemática en educación superior, particularmente en la motivación y el desempeño académico?

\section{Paso 2. Bases de datos y términos de búsqueda de literatura}

Se realizó una revisión sistemática utilizando cinco bases de datos científicas: Scopus, ISI Web of Science, ERIC, IEEE y Psyclnfo. Estas se seleccionaron por su pertinencia con el tema y por la calidad de las publicaciones que involucran investigación desde la componente educativa y tecnológica. Los tres términos principales propuestos para la revisión, los cuales se interceptan en la ecuación de búsqueda, son: 1) Aprendizaje Basado en Juegos, 2) Matemáticas y 3) Educación Superior. Cada uno de estos términos es soportado a través de sinónimos o términos clave similares, que permitan aumentar el rango de la búsqueda; así como registrados en la ecuación de búsqueda en idioma inglés. El detalle de estos términos se presenta en la Tabla 1.

Con las palabras claves mostradas en la Tabla 1 se realizaron varias iteraciones de búsqueda, analizando los resultados y la pertinencia de los mismos respecto a las preguntas de investigación de la revisión sistemática. En la ecuación 1 se presenta la búsqueda utilizada en Scopus:

( "Game based learning" OR "Game-based learning" OR edugame OR "serious game" OR "computer game" OR gamification OR "video game") AND (maths OR mathematics OR calculus OR algebra) AND ("higher education" OR university OR college OR "Tertiary education" )

Tabla 1: Términos de búsqueda utilizados en las bases de datos.

\begin{tabular}{|l|l|l|}
\hline Aprendizaje Basado en Juegos & Matemáticas & Educación Superior \\
\hline "Game based learning" & Maths & "Higher education" \\
\hline "Game-based learning" & Mathematics & University \\
\hline Edugame & Calculus & College \\
\hline "serious game" & algebra & Tertiary education \\
\hline "serious gaming" & & \\
\hline "computer game" & & \\
\hline Gamification & & \\
\hline Video game" & & \\
\hline
\end{tabular}

Cabe resaltar que la sintaxis de la ecuación fue adaptada a cada base de las cinco relacionadas previamente, así como la aplicación de los criterios de exclusión que se presentan en la siguiente sección. Luego de la unificación de los resultados de las cinco bases de datos se procedió a la depuración de los datos (eliminación de duplicados, ajuste de autores con múltiple denominación, ajuste en afiliaciones con denominaciones diferentes, entre otros). Esto se llevó a cabo utilizando el software Vantage-point. 


\section{Paso 3. Formulación de criterios de inclusión y exclusión}

Los criterios de inclusión y exclusión iniciales propuestos en la revisión fueron los siguientes: 1) periodo de la revisión: 2012-2017, 2) los tipos de documentos que se incluyeron en la revisión son artículos en revistas, documentos de conferencias y revisiones sistemáticas de la literatura. De este proceso se obtuvieron 294 registros independientes. Con este Corpus de conocimiento se procedió a realizar el análisis bibliométrico del presente artículo.

\section{Paso 4. Análisis bibliométrico de los artículos}

Como se resalta en Ceballos-Parra et al. (2018), los análisis bibliométricos proveen indicadores que permiten determinar tendencias relacionadas con un campo del conocimiento específico y la evolución que este ha tenido. Se recurre a métodos estadísticos para la identificación de autores, afiliaciones institucionales, países, entre otros aspectos. Para este paso metodológico se llevó a cabo el análisis recurriendo a la herramienta Vantage-point analizando cuatro aspectos: 1) Análisis de número de publicaciones por año, 2) Análisis de la dispersión de la bibliografía científica, 3) Análisis de publicaciones por países y 4) Análisis de publicaciones por autores.

\section{Paso 5. Análisis de la calidad científica de las publicaciones}

Para precisar aún más la revisión sistemática a las preguntas de investigación propuestas, se establecen dos criterios de exclusión adicionales. Estos son: 1) Las publicaciones deben estar asociadas a la implementación o discusión de propuestas, estrategias o acciones pedagógicas en el área de matemáticas en educación superior y 2) contar con el acceso a las versiones completas de los artículos. Se aplicaron estos criterios de exclusión dando como resultado un total de 24 artículos para proceder con una evaluación de calidad.

La calidad de los artículos se verificó utilizando 11 criterios de calidad extraídos de Petticrew y Roberts (2006) y adaptado por Gast et al. (2017) los cuales se presentan en la Tabla 2. Cada criterio se evaluó en tres escalas: 1) 0 , no está definido, 2) 0.5 , se presenta, pero no con total claridad y 3) 1 , se presenta claramente. Para ser incluidos en la revisión sistemática, los artículos debían tener una puntuación de al menos 5.5 para los 11 criterios, al menos la mitad de la cantidad máxima de puntos posible. Después de este control de calidad, quedaron 19 artículos elegibles para su inclusión en esta revisión. Ahora bien, en la Tabla 3, se presenta el listado completo de los artículos seleccionados para la revisión; así como información específica de los mismos como datos de origen, año de publicación, participantes, tipo de investigación, entre otros

Tabla 2: Criterios de calidad. (Adaptada de Gast et al., 2017).

\begin{tabular}{|c|c|}
\hline Categoría & Criterio de calidad \\
\hline \multirow[b]{2}{*}{ General } & 1. ¿Está claro el objetivo de la investigación? \\
\hline & $\begin{array}{l}\text { 2. ¿La investigación realizada con el método elegido es capaz de encontrar una } \\
\text { respuesta clara a la pregunta de investigación? }\end{array}$ \\
\hline \multirow{2}{*}{ Muestra de selección } & 3. ¿Se reunieron suficientes datos para asegurar la validez de las conclusiones? \\
\hline & 4. ¿Está claro el contexto de la investigación (país, participantes)? \\
\hline \multirow{3}{*}{ Método } & 5. ¿Indican los investigadores los métodos de investigación utilizados? \\
\hline & 6. ¿Los autores dan un argumento para los métodos elegidos? \\
\hline & $\begin{array}{l}\text { 7. ¿Los investigadores toman en cuenta otras variables que podrían ser de } \\
\text { influencia? }\end{array}$ \\
\hline \multirow{3}{*}{ Análisis de datos } & 8. ¿Se analizan los datos de manera adecuada y precisa? \\
\hline & 9. ¿Están los resultados claramente presentados? \\
\hline & $\begin{array}{l}\text { 10. ¿Los investigadores informan sobre la confiabilidad y validez de la } \\
\text { investigación? }\end{array}$ \\
\hline Conclusión & $\begin{array}{l}\text { 11. ¿Se responde a la pregunta de investigación utilizando evidencia empírica de } \\
\text { la investigación que se realizó? }\end{array}$ \\
\hline
\end{tabular}




\begin{tabular}{|c|c|c|c|c|c|c|c|c|c|}
\hline 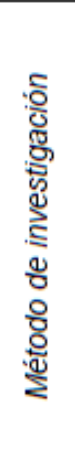 & 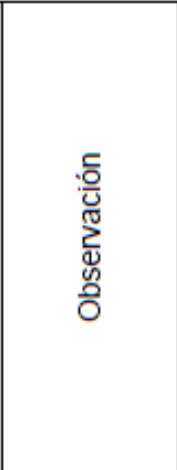 & 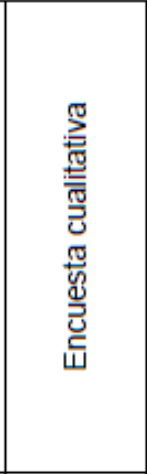 & 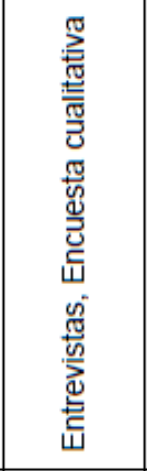 & 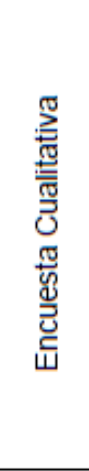 & 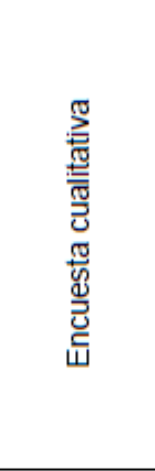 & 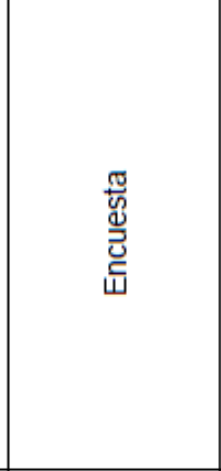 & 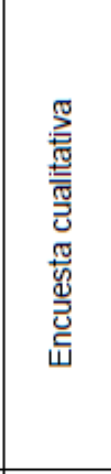 & 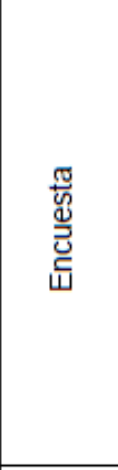 & 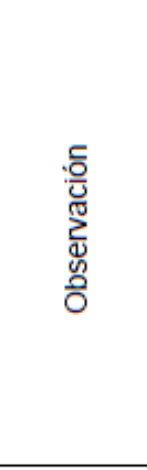 \\
\hline 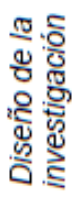 & $\frac{\pi}{\frac{\pi}{x}}$ & $\frac{\frac{\pi}{x}}{\sum}$ & 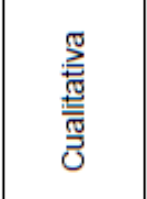 & 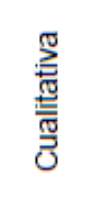 & $\frac{\sqrt[\pi]{x}}{\sum}$ & 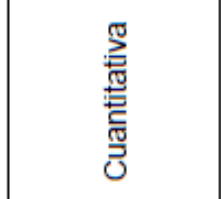 & 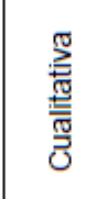 & $\frac{\sqrt[\pi]{x}}{\sum}$ & 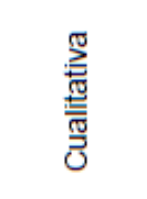 \\
\hline 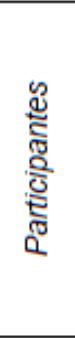 & 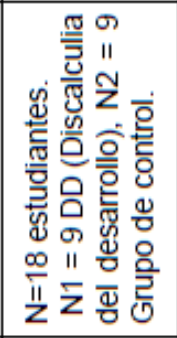 & 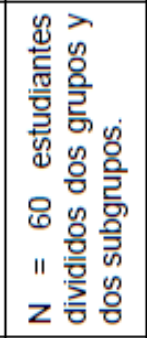 & 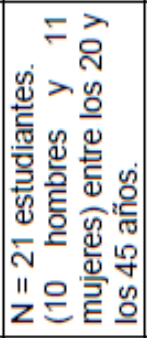 & 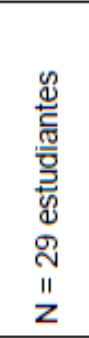 & 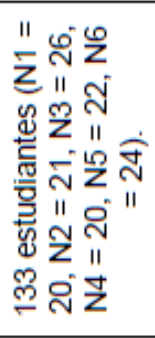 & 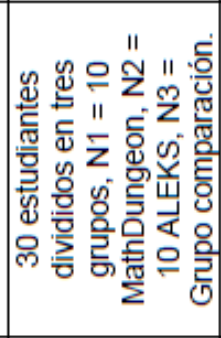 & 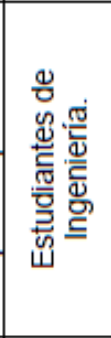 & 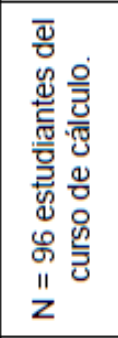 & 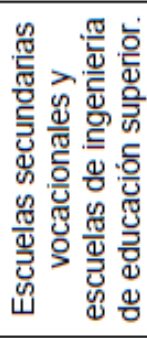 \\
\hline 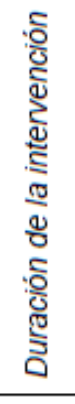 & 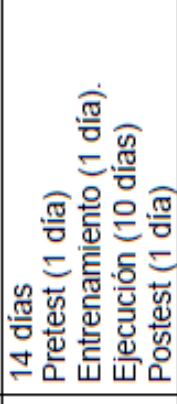 & 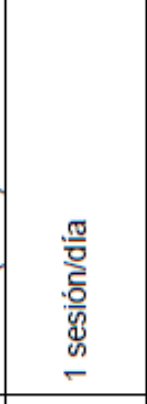 & 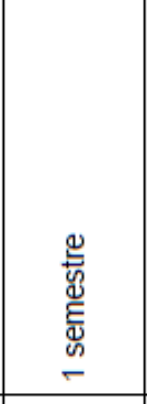 & 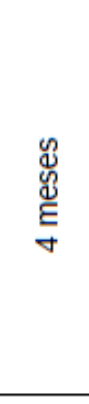 & 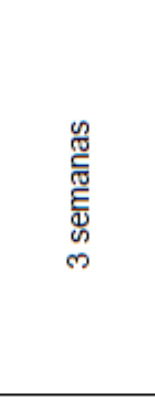 & 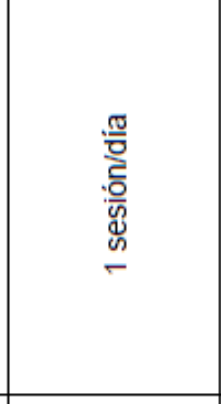 & 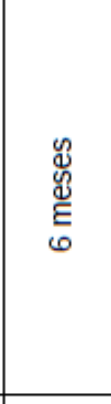 & $\stackrel{\mathscr{E}}{\underline{E}}$ & 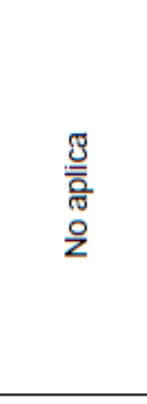 \\
\hline : & ั. & 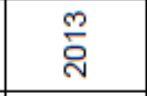 & సे & ํํํ & 호 & ন্े & $\stackrel{\llcorner 0}{\bar{c}}$ & $\stackrel{\text { Lొ }}{\bar{N}}$ & $\stackrel{\bullet}{\circ}$ \\
\hline$\frac{\mathscr{y}}{\delta}$ & $\begin{array}{l}\overline{\mathbb{W}} \\
\underline{\underline{w}}\end{array}$ & 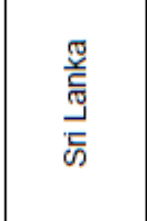 & 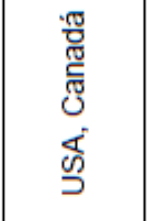 & હో & 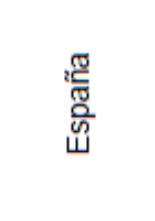 & હొ & હో & હુ & $\begin{array}{l}\frac{\pi}{0} \\
\frac{\pi}{0} \\
0\end{array}$ \\
\hline$\stackrel{\circ}{\stackrel{乛}{:}}$ & 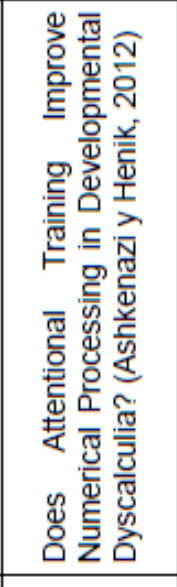 & 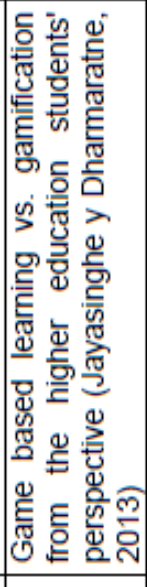 & 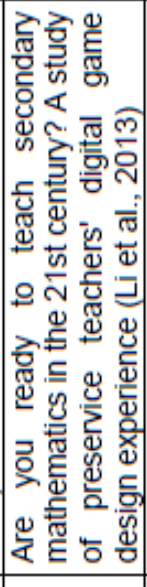 & 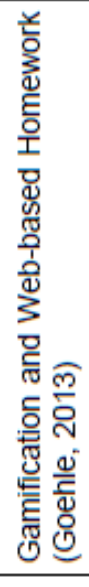 & 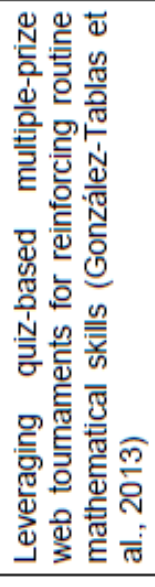 & 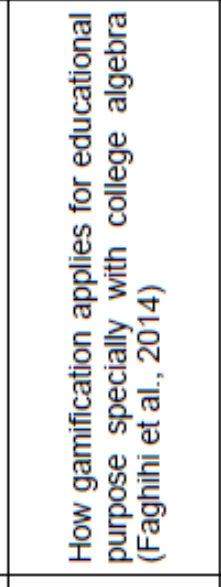 & 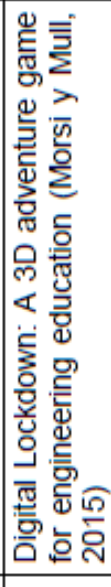 & 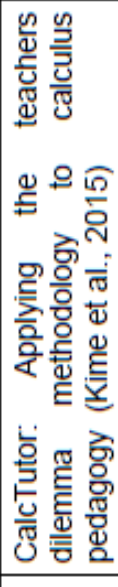 & 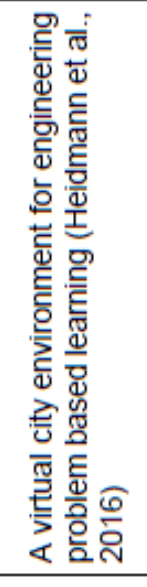 \\
\hline$\stackrel{\circ}{z}$ & - & $N$ & $m$ & $\nabla$ & is & 0 & $\lambda$ & $\infty$ & $\sigma$ \\
\hline
\end{tabular}




\begin{tabular}{|c|c|c|c|c|c|c|c|c|c|}
\hline 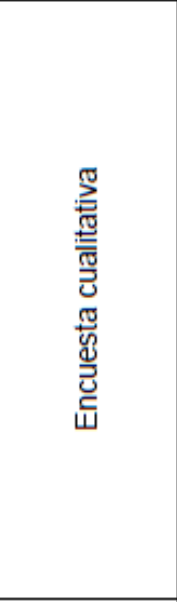 & 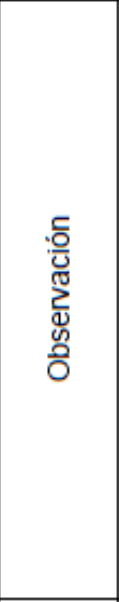 & 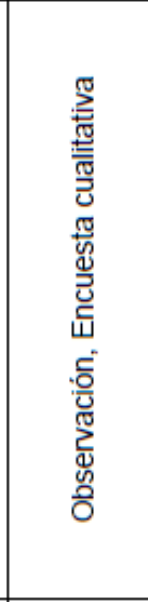 & 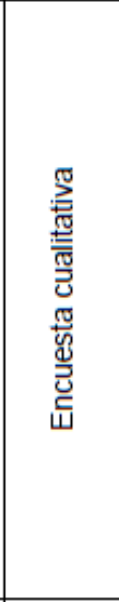 & 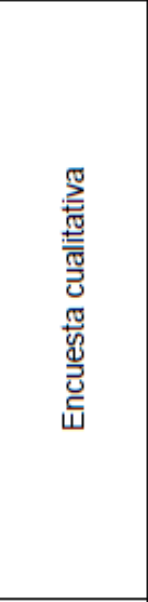 & 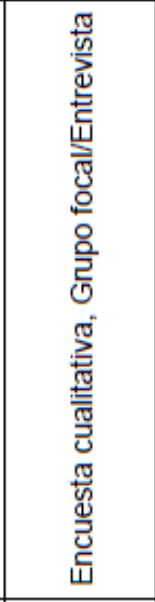 & 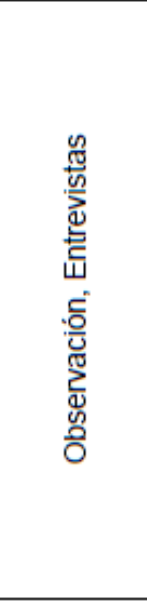 & 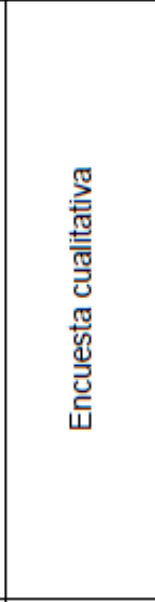 & 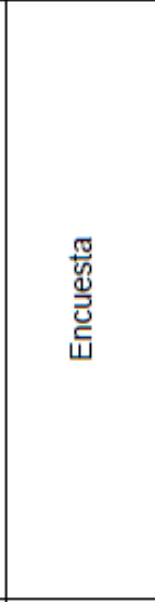 & 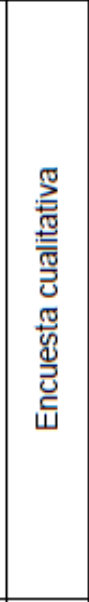 \\
\hline$\frac{\pi}{x}$ & 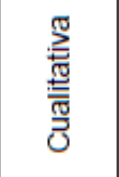 & 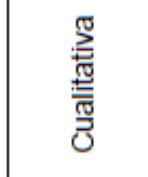 & 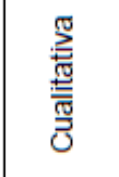 & $\frac{\sqrt{x}}{\sum}$ & 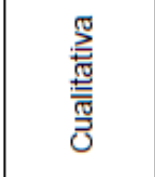 & 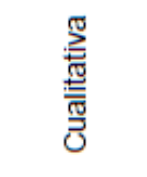 & 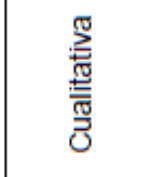 & 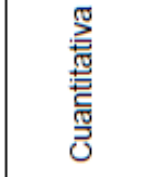 & 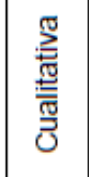 \\
\hline 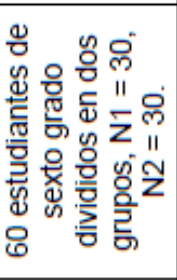 & 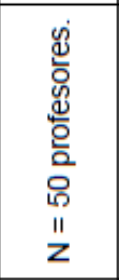 & 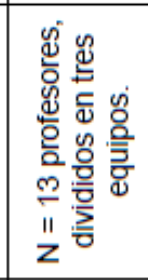 & 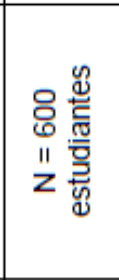 & 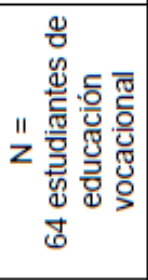 & 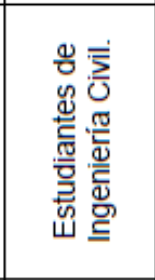 & 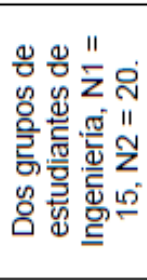 & 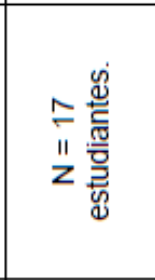 & 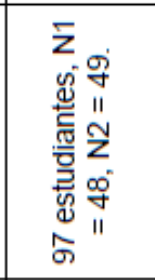 & 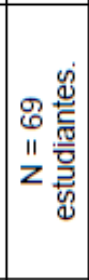 \\
\hline 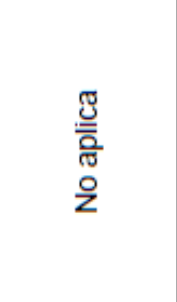 & 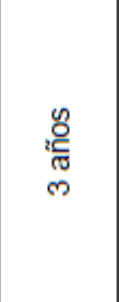 & 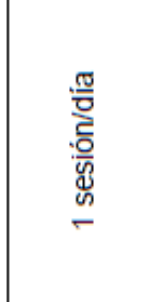 & 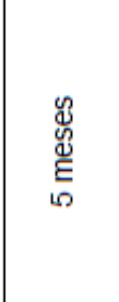 & 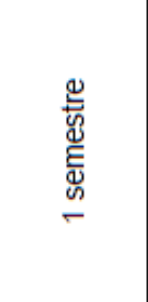 & 륨 & 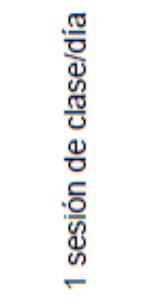 & 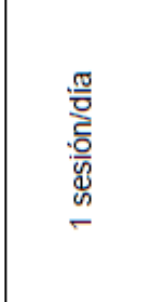 & 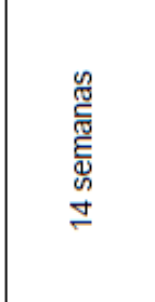 & 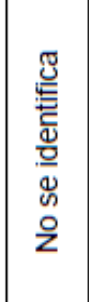 \\
\hline$\stackrel{\circ}{\circ}$ & $\stackrel{\circ}{\circ}$ & $\stackrel{\circ}{\circ}$ & $\stackrel{\circ}{\circ}$ & $\overline{\grave{n}}$ & $\overline{\hat{N}}$ & స్ & $\overline{\check{n}}$ & $\overline{\text { N }}$ & $\overline{\bar{N}}$ \\
\hline 莺 & 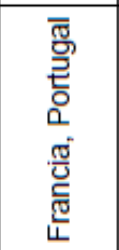 & 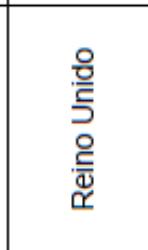 & 음 & 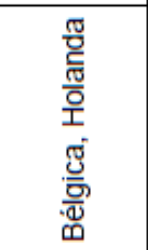 & ঙ্ & ભ & ભ & 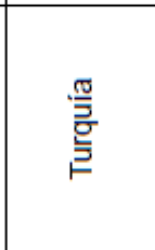 & 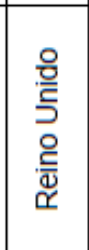 \\
\hline 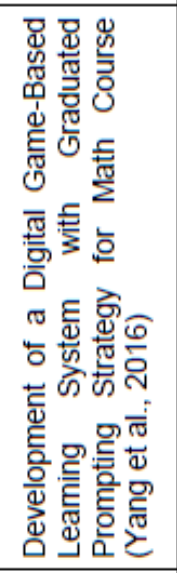 & 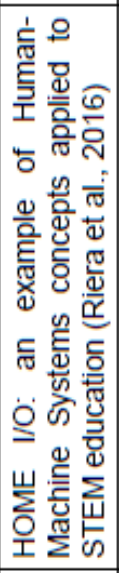 & 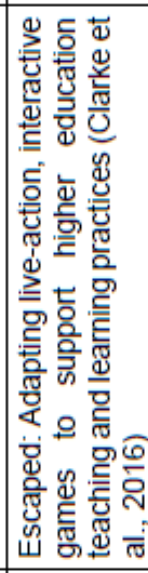 & 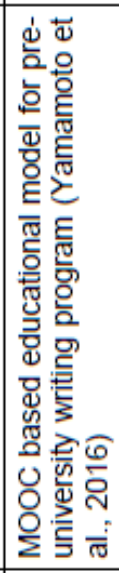 & 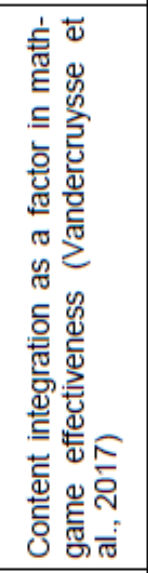 & 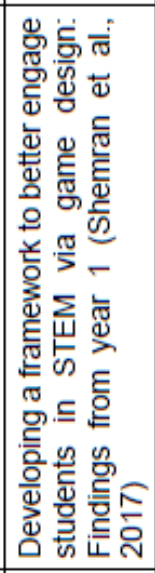 & 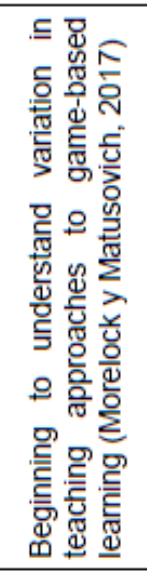 & 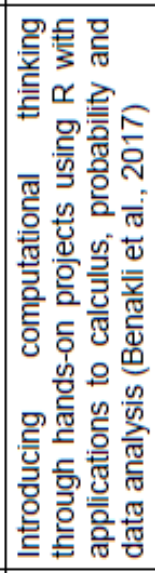 & 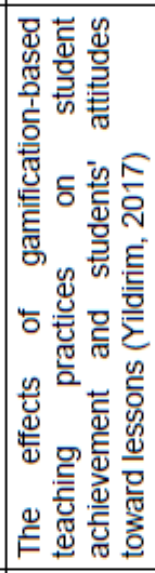 & 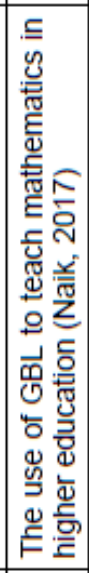 \\
\hline 으 & $\mp$ & $\cong$ & $\stackrel{m}{\longrightarrow}$ & J & $\stackrel{\leftrightarrow \Omega}{\leftarrow}$ & $\stackrel{\varphi}{\circ}$ & $\cong$ & $\stackrel{\infty}{\sim}$ & $\stackrel{\text { I }}{\leftarrow}$ \\
\hline
\end{tabular}




\section{Paso 6 - Análisis de categorías}

Este análisis se basó en la identificación de elementos que permitieron dar respuesta a las preguntas de investigación. En primera instancia (asociado a la pregunta I) se analizaron las tipologías y características de juegos, las cuales se listan a continuación: 1) el propósito primario del juego (juegos para la educación, juegos serios o entretenimiento), 2) tipo de juego (digital o no digital), 3) genero del juego (acción, rompecabezas, juegos de rol, simuladores, estrategia, aventura u otro/genérico), 4) plataforma del juego (PC/móvil en línea, PC/móvil fuera de línea, consolas de video-juegos); y 5) la subárea de las matemáticas a la cual estaba aplicado el juego (aritmética, algebra, cálculo, geometría, estadística o probabilidad). Posterior a esto (relacionado con la pregunta II), se realizó una clasificación por enfoque o diseño de investigación de las publicaciones obtenidas (cualitativa, cuantitativa y mixta); así como los instrumentos mas utilizados

Igualmente, la identificación de las salidas de interés pedagógico, que permiten dar respuesta a las dimensiones del comportamiento y el desempeño académico (asociado a la pregunta III), se centran en: 1) Dominio afectivo-motivacional, 2) dominio cognitivo de adquisición y compresión, 3) dominio comportamental y 4) dominio de habilidades blandas y habilidades sociales. Cuando se habla del dominio afectivomotivacional, se incluyen aspectos como la motivación de los estudiantes, los sentimientos y las actitudes hacia el curso, tanto intrínseca como extrínseca, así como los estímulos tanto positivos como negativos. Por su parte, el dominio cognitivo de adquisición y comprensión se refiere a aquellos procesos que se centran en las etapas de desarrollo de conocimiento y en la forma de entender saberes específicos, haciendo alusión al desempeño y rendimiento de los estudiantes en las pruebas o intentos de juego. El dominio comportamental incluye el compromiso actitudinal del estudiante con el trabajo propuesto; relacionando variables como el tiempo dedicado a una actividad, la valoración de sus aportes en las jornadas de clase o en los encuentros virtuales (según la metodología), el número y calidad de las actividades complementarias, la actitud hacia la tarea propuesta y el seguimiento a las normas propuestas. De otra parte, el dominio asociado a las habilidades blandas y habilidades sociales apunta a aquellos registros que se orienten a la observación o valoración de las competencias que permiten que los individuos se desarrollan en entornos sociales, trabajando con buen desempeño y de forma proactiva con otros (Matteson et al., 2016). Finalmente, con todos estos elementos atendidos fue posible determinar, en la discusión, la influencia del aprendizaje basado en juegos, en los procesos de enseñanza de la matemática en educación superior.

\section{RESULTADOS Y DISCUSIÓN}

Los principales resultados de la revisión se presentan a continuación, iniciando por los aspectos de análisis bibliométrico.

\section{Análisis bibliométrico}

El primer análisis que se presenta es el número de publicaciones por año. El mismo se acota entre los años 2012 y 2017, ventana temporal de la revisión. Esto es presentado en la Fig. 1. Se observa una tendencia incremental en los últimos años, resaltando que entre los años 2013 y 2014 ocurre el principal incremento en un periodo de un año. Esto se asocia coherentemente con los postulados propuestos por la Ley de incremento exponencial, aplicada por (Ceballos-Parra et al., 2018).

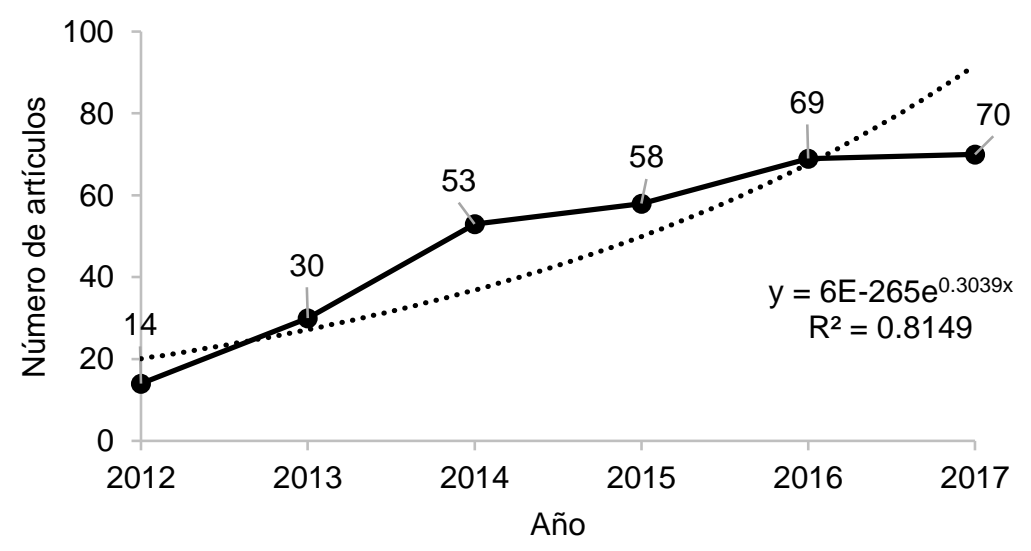

Fig. 1: Número de artículos de la revisión por año (2012-2017)

El segundo análisis bibliométrico para la revisión se asocia con la dispersión de la bibliografía científica. Según los resultados obtenidos, el $37.75 \%$ de los artículos obtenidos de la revisión en las bases de datos citadas 
(111/294) fueron publicados en revistas científicas indexadas. De estos 111 artículos, el $27,03 \%$ se concentran sólo en cinco revistas. En la Tabla 4 se relacionan estas dichas revistas y el número de registros en cada una. Cabe resaltar las primeras dos fuentes (revistas) las cuales se encuentran indexadas, según el indicador SJR de Scimago ${ }^{\circledR}$, en Cuartil 1 (Q1) con índices h de 134 y 123 respectivamente (SCIMAGO, 2018); lo que las hace de gran impacto en la comunidad científica y académica.

Tabla 4: Cinco principales revistas indexadas con publicaciones en artículos del área de la enseñanza de las matemáticas basada en juegos.

\begin{tabular}{|c|c|l|c|c|}
\hline $\begin{array}{c}\text { Tipo de } \\
\text { fuente }\end{array}$ & $\begin{array}{c}\text { No de } \\
\text { artículos }\end{array}$ & Título de la fuente & No public. \\
\hline \multirow{4}{*}{ Revistas } & \multirow{3}{*}{$\begin{array}{c}111 \\
\text { (de 294) }\end{array}$} & Computers and Education (ISSN: 0360-1315 / h index: 134) & 7 \\
\cline { 3 - 5 } & & Computers in Human Behavior (ISSN: 0747-5632 / h index: 123) & 7 \\
\cline { 3 - 5 } & & International Journal of Game-Based Learning (ISSN: 2155-6857 / h index: 11) & 6 \\
\cline { 3 - 5 } & & Technology, Knowledge and Learning (ISSN: 2211-1670/ h index: 11) & 5 \\
\cline { 3 - 5 } & & Interactive Learning Environments (ISSN: 1049-4820 / h index: 31) & 5 \\
\hline
\end{tabular}

Otro de los aspectos que se analizaron fue la relación de publicaciones por países, con el fin de identificar los países con mayor productividad en el campo de la enseñanza de las matemáticas basada en juegos-GBL. Como resultado, se identificaron 53 países investigando en el campo de estudio. Los 5 países más productivos fueron: Estados Unidos (USA), China, Reino Unido, Grecia y España; con 86, 22, 21,18 y 15 contribuciones respectivamente. El primer país Latinoamericano en la lista es Brasil (puesto 12) con 7 publicaciones. Los primeros 15 países por cantidad de publicaciones se presentan en la Fig. 2.

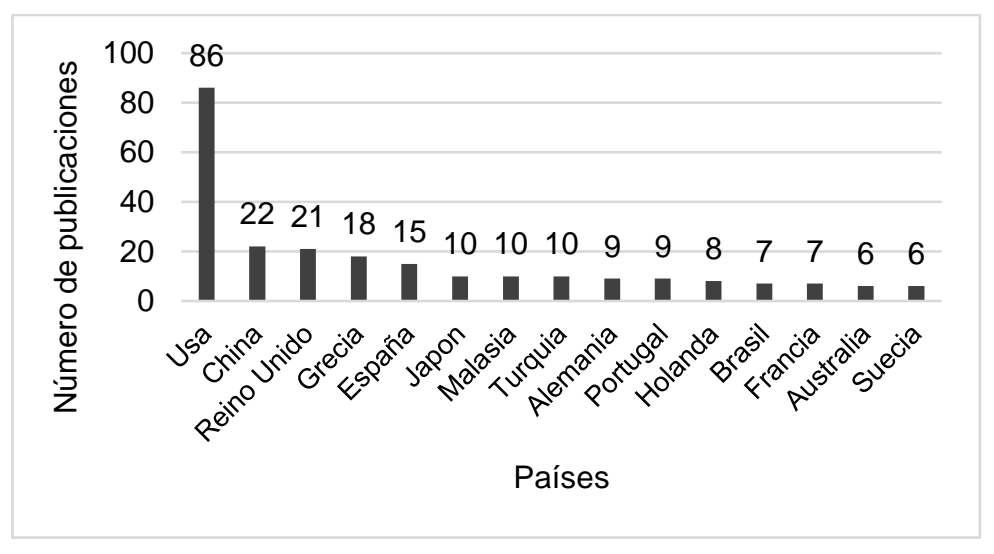

Fig. 2: Relación de número de publicaciones por países (primeros 15)

En cuanto a los autores que más contribuciones hacen en el tema de la presente revisión, es posible citar a los mostrados en la Tabla 5. No se encuentra un autor con más de 10 publicaciones en el tema, y según los datos obtenidos existe una gran dispersión en el tema. De las 294 publicaciones se relacionan 886 autores diferentes, de los cuales solo 86 cuentan con dos o más publicaciones.

Tabla 5: Autores con mayor cantidad de publicaciones en la revisión realizada

\begin{tabular}{|l|c|}
\hline Autor & № public. \\
\hline Ahmad, M. & 5 \\
\hline Hwang, Gwo-Jen & 5 \\
\hline Ke, Fengfeng & 5 \\
\hline Arshad, N.I. & 4 \\
\hline Naik, N & 4 \\
\hline Rahim, L. A. & 4 \\
\hline Van Oostendorp, Herre & 4 \\
\hline Wouters, Pieter & 4 \\
\hline
\end{tabular}

Análisis de Categorías de acuerdo con los hallazgos en las publicaciones.

En esta sección, se presentan los hallazgos que permiten dar respuesta a las preguntas de investigación. Este análisis se ha realizado según la categorización compartida en el paso 6 de la metodología. En la Tabla 6 se relaciona la categorización según las variables del juego, buscando dar respuesta a la pregunta I. 
Tabla 6: Categorías según las variables del juego vs artículos asociados.

\begin{tabular}{|c|c|}
\hline Categorías & Artículos \\
\hline \multicolumn{2}{|l|}{ Propósito primario del juego } \\
\hline Juegos para la educación & $1,2,3,4,5,6,7,8,9,10,12,14,15,16,17,18,19$ \\
\hline Juegos serios & 11,13 \\
\hline Entretenimiento & Ninguno \\
\hline \multicolumn{2}{|l|}{ Digital/No digital } \\
\hline Digital & $1,2,4,5,7,8,9,11,12,13,14,15,16,17,18$ \\
\hline No digital & $6,10,19$ \\
\hline Digital y No digital & 3 \\
\hline \multicolumn{2}{|l|}{ Género del Juego } \\
\hline Rompecabezas & 10,19 \\
\hline Juegos rol & 2,6 \\
\hline Aventura & $3,5,8,11$ \\
\hline Otro/Genérico & 6,16 \\
\hline Simulador & $1,13,15$ \\
\hline $\begin{array}{l}\text { Plataforma virtual de enseñanza - } \\
\text { Sistemas de Gestión de Aprendizaje } \\
\text { (LMS) }\end{array}$ & $4,7,9,12,14,17,18$ \\
\hline \multicolumn{2}{|l|}{ Plataforma } \\
\hline PC/móvil fuera de línea & $1,3,15$ \\
\hline Consola de video juegos & 13 \\
\hline PC/móvil en línea & $2,4,5,7,8,9,11,12,14,16,17,18$ \\
\hline \multicolumn{2}{|l|}{ Sub-área de matemáticas } \\
\hline Aritmética & $5,7,9,16,17,18$ \\
\hline Álgebra & $2,8,14$ \\
\hline Probabilidad & 15 \\
\hline Cálculo & $4,12,15$ \\
\hline
\end{tabular}

En cuanto a la categoría de Propósito primario del juego, existen 17 artículos que plantearon juegos que desde su orientación inicial (diseño e implementación) ha sido la educación. Los restantes 2 artículos del total de registros abordan juegos serios. El propuesto por Riera et al. (2016) diseña un juego llamado HOME I/O, basado en un software de simulación en tiempo real de una casa inteligente para generar conciencia en la eficiencia energética. De otra parte, el segundo es el propuesto por Yamamoto et al. (2016), donde se implementa un conjunto de estrategias pedagógicas donde, una de estas, es la herramienta para evaluación denominada Hot Potatoe (que puede ser considerado un juego serio por su aplicación en entornos organizacionales). Finalmente, en la revisión realizada no se encuentra ningún registro cuya intervención se haya realizado con un aplicativo software con orientación primaria de entretenimiento.

En la categoría de Juegos de naturaleza digital o no digital se observa que 15 de los estudios se centraron en la primera opción (digitales). La búsqueda identificó 4 artículos que implementaron juegos no digitales. Morelock y Matusovich (2017) en su investigación, abordaron cómo los instructores de ingeniería usan los juegos en el aula y cómo estas prácticas de enseñanza se relacionan con la motivación de los estudiantes para aprender. Por otra parte, Shemran et al. (2017) desarrollaron un marco para involucrar mejor a los estudiantes en el desarrollo de habilidades en Ciencia, Tecnología, Ingeniería y Matemáticas (STEM) a través del diseño de juegos de mesa, como un método para evaluar el dominio de los estudiantes en los conceptos de construcción e ingeniería. Por su parte, el trabajo realizado por Clarke et al. (2016) se enfocó en el programa titulado EscapED. El propósito de este es conceptualizar experiencias interactivas y ayudar a otros facilitadores educativos a crear sus propios juegos de acción en vivo con fines educativos y cambios positivos de comportamiento en entornos de educación superior. Finalmente, Naik (2017) diseñó cuatro formas de juegos no digitales orientados a: 1) aritmética - fracciones rummy, 2) un crucigrama decimal, 3) un rompecabezas logarítmico y exponencial y 4) un bingo de ecuaciones algebraicas.

Respecto a la categoría de Género de juego es posible afirmar que los juegos en plataformas virtuales de enseñanza o Sistemas de Gestión de Aprendizaje (LMS) son el género más popular con 7 publicaciones, seguido de los juegos aventuras con 4, los simuladores y otro/genérico fueron soportados por 3 artículos paralelamente. Los rompecabezas (Puzzles) tuvieron el soporte de 2 artículos, mientras que hubo solo una publicación que soporte el género de juego de rol. Diecisiete estudios examinaron el impacto de los juegos en general, mientras que dos esperan evaluar este impacto en el futuro. En el mismo sentido, 2 estudios clasificaron su juego en el género otro/genérico: Shemran et al. (2017) exploran el uso de juegos de mesa desarrollados por los estudiantes como un método para evaluar el dominio de los estudiantes en los conceptos 
de construcción e ingeniería. Por su parte, González-Tablas (2013), buscó reforzar las habilidades matemáticas de rutina con un sistema de torneos web. El juego está diseñado como un torneo basado en un concurso con una estructura de múltiples premios y rangos de puntuación pública, con el propósito de disminuir las desventajas de las competencias interpersonales puras y motivar la participación de los estudiantes.

Sobre la categoría de plataforma más popular para la ejecución de los juegos fue Computador (PC)/móvil en línea (12), seguida de Computador (PC)/móvil fuera de línea (3) y la consola de video juegos (1). De las 12 publicaciones que se distribuyeron en PC/móvil en línea, 7 fueron plataformas virtuales de enseñanza, 3 juegos de aventura, 1 juego de rol y 1 clasificado en otro/genérico. De los distribuidos en PC/móvil fuera de línea, 2 fueron simuladores y 1 de aventura. El único que se desarrolló por medio de consola de video juegos fue un simulador. En cuanto a las subáreas de las matemáticas, 6 de los juegos se utilizaron para el aprendizaje de la aritmética, 3 estuvieron orientados al álgebra y 3 al cálculo equitativamente. Es importante mencionar que Benakli et al. (2017), en su artículo planteó nueve experimentos con aplicaciones para el cálculo, la probabilidad y el análisis de datos. Es por eso por lo que tuvo una doble clasificación. Sin embargo, existieron publicaciones donde no estaba disponible o no fue clara la información de la subárea a la cual estaba orientado el juego, como fue el caso de: (Benakli et al., 2017; Clarke et al., 2016; Goehle, 2013; Heidmann et al., 2016; Morelock y Matusovich, 2017; Riera et al., 2016; Shemran et al., 2017; Yildirim, 2017). Ahora bien, respecto a la pregunta II (metodologías e instrumentos), es posible afirmar que el $58 \%$ de los registros utilizan un enfoque investigación de corte cualitativo, siendo la más utilizada en el corpus de conocimiento revisado. Un $32 \%$ aborda enfoque mixto y solo el $11 \%$ recurre a la ruta cuantitativa como enfoque de investigación. Respecto a los instrumentos utilizados, el más popular en los artículos revisados en la Encuesta cualitativa, apareciendo en cerca del 63\%; seguido de la Observación (21\%), la Entrevista (16\%) y la Encuesta (16\%). Cabe citar que el total de la adición de los porcentajes relacionados supera el $100 \%$ pues existen investigaciones que relacionan más de un instrumento.

En la Tabla 7 se presentan los artículos según las categorías asociadas a las salidas del proceso o resultados de aprendizaje. Con estos elementos es posible argumenta sobre la pregunta III (Dimensiones del comportamiento y desempeño de los estudiantes); ya que la clasificación expone el abordaje de los diferentes artículos a cada uno de los dominios previamente establecidos.

Tabla 7: Categorías según los resultados de aprendizaje o salidas del proceso vs artículos asociados.

\begin{tabular}{|l|l|}
\hline Categorías & Artículos \\
\hline Dominio cognitivo de adquisición y comprensión & $1,2,3,4,5,6,7,8,9,10,11,12,13,14,15,16,17,18,19$ \\
\hline Dominio afectivo-motivacional & $3,4,5,8,9,10,11,12,14,15,16,17,18,19$ \\
\hline Dominio comportamental & $4,10,15,17$ \\
\hline $\begin{array}{l}\text { Dominio de habilidades blandas y habilidades } \\
\text { sociales }\end{array}$ & $8,9,12,13,15,17$ \\
\hline
\end{tabular}

En cuanto al dominio cognitivo de adquisición y comprensión del aprendizaje, todos los estudios resaltan interés o consideración del aspecto. Esto es una característica importante al evaluar la orientación de dichas investigaciones y el interés de la comunidad académica en la relación entre el uso de juegos y el impacto directo al desarrollo de conocimiento. Muchos de estos estudios demuestran que el uso de los juegos afectan, de forma positiva, el desarrollo de habilidades y competencias en la población objetivo, lo cual es coincidente con lo discutido por (Hamari et al., 2014). El segundo dominio de mayor interés para las investigaciones es el asociado al plano de la motivación. Varios de los estudios no lo refieren como una variable de interés directa, no obstante, se observa una fuerte relación con los procesos del dominio cognitivo y el afectivo-emocional. Por ejemplo, Heidmann et al. (2016) y Morelock y Matusovich (2017), exclusivamente examinaron el impacto del juego en la participación y motivación de los estudiantes; Kime et al. (2015), Morsi y Mull (2015), Clarke et al. (2016) y Goehle (2013) evaluaron la adquisición y desarrollo de conocimientos determinando el desempeño y el rendimiento de los estudiantes una vez participaron en el juego. Los resultados arrojaron un impacto positivo en la motivación y en el rendimiento de los estudiantes. Como cita (Connolly et al., 2012) el desarrollo de este dominio genera, en muchos casos, un aprendizaje no intencional, lo cual genera finalmente un fomento en el compromiso de los estudiantes al proceso educativo.

Un tercer elemento para considerar es el Dominio de habilidades o competencias blandas y sociales. Este tipo de resultados aparecen en seis (6) de los registros analizados, sin que sean de análisis directo o intencionado, es decir que emergen en la búsqueda de resultados de aprendizaje específicos; lo que se observa claramente en los trabajos de (Benakli et al., 2017; Clarke et al., 2016; Heidmann et al., 2016; Kime et al., 2015; Shemran et al., 2017; Yamamoto et al., 2016). Finalmente, el Dominio comportamental es el que tiene menos estudios asociados (4), y al igual que en el caso anterior no se evidencia como intencionalidad primaria de las investigaciones. Se resalta el trabajo presentado por Goehle (2013), donde los estudiantes, 
de forma autónoma, realizan el monitoreo de sus acciones formativo e intentan voluntariamente autorregular el desarrollo de los logros y su comportamiento hacia el proceso educativo. Este último aspecto se encuentra acorde con parte de los resultados y discusiones propuestos en (Boyle et al., 2016).

\section{CONCLUSIONES}

La presente revisión bibliográfica permite identificar una tendencia incremental en la publicación, resultado de intereses de investigación, del uso del aprendizaje basado en juegos en educación superior, particularmente para la enseñanza de la matemática. Los tres países con mayor índice de publicación en el área son Estados Unidos de América, China y Reino Unido. Las publicaciones con mayor cantidad de artículos en el área son Computer and Education, Computer in Human Behavior y el International Journal of Game-Based Learning. En la gran mayoría de los estudios revisados las características asociadas a la mediación o artefacto de apoyo (juego) han sido diseñados con el propósito específico de la educación.

Los juegos digitales priman en los registros observados, sin embargo, también se evidencia la existencia del uso de los juegos no digitales (tradicionales). El mayor porcentaje de herramientas tecnológicas se han implementado en plataformas digitales en línea y la subárea de la matemática más revisada es la aritmética. Las salidas o resultados de aprendizaje de las experiencias analizadas se centran, en su gran mayoría, en el fomento de procesos cognitivos, es decir asociados a la adquisición de competencias y habilidades; así como a la comprensión de conceptos. Estos aspectos son claramente intencionados en las investigaciones revisadas. El dominio afectivo y emocional se encuentra también en un alto porcentaje de los estudios, con procesos de investigación que se orientan a fortalecer el compromiso de los estudiantes en los procesos de aprendizaje, la relación de lo que se aprende con su contexto e intereses, así como en sus metas personales.

Finalmente, la revisión sistemática realizada permite observar que el uso de los juegos en los procesos de enseñanza-aprendizaje, mantiene gran relevancia e interés en la comunidad académica y científica. Asimismo, que cuenta con un gran potencial para la formación en matemáticas de educación superior.

\section{AGRADECIMIENTOS}

Los autores del presente artículo agradecen el aporte del Lic. César Acevedo-Argüello, de la Unidad de Bibliometría del Centro de Recursos para el Aprendizaje y la Investigación CRAl; y de los docentes del Departamento de Ciencias Básicas en el área de matemáticas: Magister Edgar Arciniegas-Hernández y PhD. Jerson Reina-Medrado, de la Universidad Santo Tomás- Seccional Bucaramanga. Se extiende también el agradecimiento al programa de Doctorado en Tecnología Educativa de la Universidad de las Islas BalearesEspaña.

\section{REFERENCIAS}

ABET, Criteria for Accrediting Engineering Programs (en la web: https://bit.ly/2Z0G0B6, acceso: 1 de Marzo de 2006), ABET, USA (2016).

Ashkenazi, S. y A. Henik, Does Attentional Training Improve Numerical Processing In Developmental Dyscalculia?, doi: 10.1037/a0026209, Neuropsychology, 26(1), 45-56 (2012).

Barquero, B.; M. Bosch y J. Gascón, Los Recorridos de Estudio e Investigación y la Modelización Matemática en la Enseñanza Universitaria de las Ciencias Experimentales. Enseñanza de las Ciencias, 29(3), 339-352 (2011).

Benakli, N.; B. Kostadinov; A. Satyanarayana y S. Singh, Introducing Computational Thinking Through Hands-On Projects using $R$ with Applications to Calculus, Probability and Data Analysis, International Journal of Mathematical Education in Science \& Technology, 48(3), 393-427 (2017).

Bergeson, T., Teaching and Learning Mathematics. Using Research to Shift From the "Yesterday" Mind to the "Tomorrow" Mind, 1a edición, 1-108, Olympia, USA (2000).

Blohm, I. y J. Leimeister, Gamification, doi: 10.1007/s12599-013-0273-5, Business \& Information Systems Engineering, 5(4), 275-278 (2013).

Borras, O.; M. Martínez y A. Blanco, Gamification in MOOC: Challenges, Opportunities and Proposals for Advancing MOOC Model; Proceedings of the Second International Conference on Technological Ecosystems for Enhancing Multiculturality, 215220, Salamanca-España, 1-3 de Octubre (2014).

Boyle, E. A.; T. Hainey y otros 7 autores, An Update to the Systematic Literature Review of Empirical Evidence of the Impacts and Outcomes of Computer Games and Serious Games, doi: 10.1016/j.compedu.2015.11.003, Computers \& Education, 94(Supplement C), 178-192 (2016).

Cabero-Almenara, J., Tendencias Educativas para el siglo XXI, 1a edición,1-95, Madrid, España (2016). 
Ceballos-Parra, P.J.; W. A. Sarache y D.M. Gómez, Un Análisis Bibliométrico de las Tendencias en Logística Humanitaria, doi: 10.4067/S0718-07642018000100091, Información Tecnológica, 29(1), 91-104 (2018).

Chen, M.P. y L.C. Wang, The Effects of Type of Interactivity in Experiential Game-Based Learning; In Learning by Playing. Game-based Education System Design and Development, 1a edición, Springer, pp 273-282, Berlin, Alemania (2009).

Clarke, S.; S. Arnab y otros tres autores, EscapED: Adapting Live-Action, Interactive Games to Support Higher Education Teaching and Learning Practices; Games and Learning Alliance, 1a edición, Springer, pp. 144-153, Berlin, Alemania (2016).

Collazos, C.; C. González y R. García, Computer Supported Collaborative MOOCs: CSCM; Proceedings of the 2014 Workshop on Interaction Design in Educational Environments, 28-32, Albacete-España, 19 de Junio (2014).

Connolly, T.M.; E.A. Boyle y otros tres autores, A Systematic Literature Review of Empirical Evidence on Computer Games and Serious Games, doi: 10.1016/j.compedu.2012.03.004, Computers \& Education, 59(2), 661-686 (2012).

Deterding, S.; D. Dixon; R. Khaled y L. Nacke, From Game Design Elements to Gamefulness: Defining "Gamification."; Proceedings of the 15th International Academic MindTrek Conference: Envisioning Future Media Environments, 9-15, New York-USA, 28-30 de Septiembre (2011).

ENAEE, EUR-ACE® Framework Standards and Guidelines, European Network for Engineering Accreditation (2015).

Faghihi, U.; A. Brautigam y otros 5 autores, How Gamification Applies for Educational Purpose Specially with College Algebra, doi: 10.1016/j.procs.2014.11.102, Procedia Computer Science, 41, 182-187 (2014).

Farias, D. y J. Pérez, Motivación en la Enseñanza de las Matemáticas y la Administración, doi: 10.4067/S071850062010000600005, Formación Universitaria, 3(6), 33-40 (2010).

Fonseca-Tovar; L., C. Suarez-Gasca y S. Zabala-Vargas, Estrategias Pedagógicas, Fundamentadas en Aprendizaje Basado en Juegos, para el Mejoramiento en el Proceso de Enseñanza del Concepto de Fracciones Matemáticas, en Estudiantes de Último Nivel de Básica Primaria, Las Competencias y la Sociedad del Conocimiento, 1a Edición, CIMTED, pp 130-154, Medellín, Colombia (2018).

Galbis-Córdova, A.; J. Martí-Parreño y R. Currás-Pérez, Higher education students' attitude towards the use of gamification for competencies development, Journal of e-Learning and Knowledge Society, 13(1), 129-146 (2017).

Gast, I.; K. Schildkamp y J.T. van der Veen, Team-Based Professional Development Interventions in Higher Education: $A$ Systematic Review, doi: /10.3102/0034654317704306, Review of Educational Research, 87(4), 736-767 (2017).

Goehle, G., Gamification and Web-based Homework. Primus: Problems, Resources \& Issues in Mathematics Undergraduate Studies, 23(3), 234-246 (2013).

González-Tablas, A.I.; J.M. de Fuentes; J.L. Hernández-Ardieta y B. Ramos. Leveraging Quiz-based Multiple-prize Web Tournaments for Reinforcing Routine Mathematical Skills,Journal of Educational Technology \& Society, 16(3), 28-43 (2013).

Hamari, J.; J. Koivisto y H. Sarsa, Does Gamification Work? -- A Literature Review of Empirical Studies on Gamification; 2014 47th Hawaii International Conference on System Sciences, 3025-3034, Hawaii-Usa, 6-9 de Enero (2014).

Heidmann, O.; C. Vaz de Carvalho y otros tres autores, A Virtual City Environment for Engineering Problem Based Learning; International Conference on Serious Games, Interaction, and Simulation, 74-79, Porto-Portugal, 16-17 de Junio (2016).

Hernandez-Horta, I.A., A. Monroy-Reza y M. Jimenez-García, Aprendizaje Mediante Juegos Basados en Principios de Gamificación en Instituciones de Educación Superior, doi: 10.4067/S0718-50062018000500031, Formación Universitaria, 11(5), 31-40 (2018).

Huang, W.D.; T.E. Johnson y S.-H.C Han, Impact of Online Instructional Game Features fn College Students' Perceived Motivational Support and Cognitive Investment: A Structural Equation Modeling Study, doi: 10.1016/j.iheduc.2012.11.004, The Internet and Higher Education, 17, 58-68 (2013).

Jayasinghe, U. y A. Dharmaratne, Game Based Learning vs. Gamification from the Higher Education Students' Perspective; Proceedings of 2013 IEEE International Conference on Teaching, Assessment and Learning for Engineering (TALE), 683-688, Bali-Indonesia, 26-29 de Agosto (2013).

Johnson, D.; R. Johnson y E. Holubec, Cooperative Learning in the Classroom, 1ํㅡ ed.,1-146, Assn for Supervision \& Curriculum Editor, Alexandría-USA (1994).

Kapp, K., The Gamification of Learning and Instruction: Game-Based Methods and Strategies for Training and Education, $1^{\text {a }}$ ed, 336, Wiley, San Francisco-USA (2012).

Ke, F. y Y.-C. Hsu, Mobile Augmented-Reality Artifact Creation as a Component of Mobile Computer-Supported Collaborative Learning, doi: 10.1016/j.iheduc.2015.04.003, The Internet and Higher Education, 26, 33-41 (2015).

Kebritchi, M.; A. Hirumi y H. Bai, The Effects of Modern Mathematics Computer Games on Mathematics Achievement and Class Motivation, doi: 10.1016/j.compedu.2010.02.007, Computers \& Education, 55(2), 427-443 (2010).

Kime, K.; R. Torrey y T. Hickey, CalcTutor: Applying the Teachers Dilemma Methodology to Calculus Pedagogy; 2015 IEEE Frontiers in Education Conference (FIE), 1-8, Texas-Usa, 21-24 de Octubre (2015).

Li, Q.; C. Lemieux; E. Vandermeiden y S. Nathoo, Are You Ready to Teach Secondary Mathematics in the 21st Century? A Study of Preservice Teachers' Digital Game Design Experience, Journal of Research on Technology in Education, 45(4), 309-337 (2013). 
Lizcano-Dallos, A.; J. Parra-Valencia y E. Pineda-Ballesteros, Categorías Ontológicas en el Aprendizaje Colaborativo: La Solución de Casos Matemáticos, Revista Virtual Universidad Católica Del Norte, 48, 100-115 (2016).

Martínez, G.A., Diseño de una Guía Didáctica basada en la Integración de Mundos Virtuales al Entorno Educativo de la Universidad de Cundinamarca, doi: 10.4067/S0718-50062017000100002, Formación Universitaria, 10(1), 3-14 (2017).

Matteson, M.L.; L. Anderson y C. Boyden, "Soft Skills": A Phrase in Search of Meaning, doi: 10.1353/pla.2016.0009, Libraries and the Academy, 16(1), 71-88 (2016).

Ministerio de Educación Nacional de Colombia, Resolución Número 2773 de 2013, Bogotá-Colombia (2003).

Morelock, J.R. y H.M. Matusovich, Beginning to Understand Variation in Teaching Approaches to Game-Based Learning; 2017 IEEE Frontiers in Education Conference (FIE), 1-5, Indianapolis-Usa, 18-21 de Octubre (2017).

Morsi, R. y S. Mull, Digital Lockdown: A 3D Adventure Game for Engineering Education. 2015 IEEE Frontiers in Education Conference (FIE), 1-4, Texas-Usa, 21-24 de Octubre (2015).

Nah, F.F.-H.; Q. Zeng y otros tres autores, Gamification of Education: A Review of Literature; HCl in Business: First International Conference, HCIB 2014, Held as Part of HCl International 2014, 401-409, Heraklion, Crete, Greece, $22-27$ de Junio (2014).

Naik, N, The use of GBL to Teach Mathematics in Higher Education, doi: 10.1080/14703297.2015.1108857, Innovations in Education and Teaching International, 54(3), 238-246 (2017).

Olmedo, V.H. y R. Ariza, Matemáticas en Medicina: una Necesidad de Capacitación, Medicina Interna de México, 28, 278281 (2012).

Petticrew, M. y H. Roberts, Systematic Reviews in the Social Sciences: A Practical Guide. 265-276, Oxford, England: Blackwell (2006).

Riera, B.; D. Annebicque y B. Vigário, HOME I/O: an Example of Human-Machine Systems Concepts Applied to STEM Education, doi: 10.1016/j.ifacol.2016.10.530, IFAC-PapersOnLine, 49(19), 233-238 (2016).

Salinas, J.; B. de Benito y A. Lizana, Competencias Docentes para los Nuevos Escenarios de Aprendizaje, https://www.redalyc.org/articulo.oa?id=27431190010, ISSN: 0213-8646, Revista Interuniversitaria de Formación del Profesorado, 28(1), 145-163 (2014).

SCIMAGO, Scimago Journal \& Country Rank, Granada-España (2018).

Shemran, R.P.; R.M. Clark y otros tres autores, Developing a Framework to Better Engage students in STEM via Game Design: Findings from Year 1; 2017 ASEE Annual Conference and Exposition, Columbus-Usa, 25-28 de Junio (2017).

Steiner, C.M.; M.D Kickmeier-Rust y D. Albert, Little Big Difference: Gender Aspects and Gender-Based Adaptation in Educational Games; Learning by Playing. Game-based Education System Design and Development, pp 150-161, Berlin, Heidelberg: Springer Berlin Heidelberg (2009).

Subhash, S. y E.A. Cudney, Gamified Learning in Higher Education: a Systematic Review of the Literature, doi: 10.1016/j.chb.2018.05.028, Computers in Human Behavior, 87, 192-206 (2018).

Sung, H.-Y., G.-J. Hwang y Y.-F. Yen, Development of a Contextual Decision-Making Game for Improving Students' Learning Performance in a Health Education Course, doi: 10.1016/j.compedu.2014.11.012, Computers \& Education, 82, 179-190 (2015).

Vandercruysse, S.; J. ter Vrugte y otros cinco autores, Content Integration as a Factor in Math-game Effectiveness, doi: 10.1007/s11423-017-9530-5, Educational Technology Research and Development, 65(5), 1345-1368 (2017).

Yamamoto, T.; T. Sasaki y S. Hayashida, MOOC Based Educational Mo del for Pre-University Writing Program, International Symposium on Grids and Clouds 2015, 13, Taiwan-China, 15-20 de Marzo (2016).

Yang, K.; J. Chen y B. Lu, Development of a Digital Game-Based Learning System with Graduated Prompting Strategy for Math Course; 2016 5th IIAI International Congress on Advanced Applied Informatics (IIAI-AAI), 423-426, Kumamoto-Japón, 10-14 de Julio (2016).

Yildirim, I, The Effects of Gamification-Based Teaching Practices on Student Achievement and Students' Attitudes Toward Lessons, doi: 10.1016/j.iheduc.2017.02.002, The Internet and Higher Education, 33, 86-92 (2017).

Zabala-Vargas, S.; L. Montenegro y D. Alfonso, Representación Computacional y Desarrollo de la Competencia de Modelamiento, El modelamiento matemático en la formación del ingeniero, 1a Edición, pp 149-171, Universidad Central, Bogotá-Colombia (2013). 